\title{
INFLUENCE OF THE SUBSTRATE STRUCTURE ON CARBOXYPEPTIDASE Y CATALYZED PEPTIDE BOND FORMATION
}

\author{
by \\ KLAUS BREDDAM, FRED WIDMER and JACK T. JOHANSEN \\ Department of Chemistry, Carlsberg Laboratory, \\ Gamle Carlsberg Vej 10, DK-2500 Copenhagen Valby
}

Keywords: Enzymatic synthesis, peptide synthesis, carboxypeptidase Y

It is shown that for carboxypeptidase $\mathrm{Y}$ catalyzed peptide synthesis the coupling yields using peptide esters as substrates and amino acids as nucleophiles are strongly dependent on the C-terminal amino acid residue. A dependence on the length of the acyl donating peptide chain is also observed, indicating the influence of residues other than the one directly involved in acylation of the enzyme. Using amino acid amides as nucleophiles, the yields are generally high $(80 \%-100 \%)$. No peptide ester substrate with C-terminal D-amino acid residue can be used in this method of peptide synthesis since they are not substrates of the enzyme. However, the presence of Damino acids in the penultimate position does not prevent peptide synthesis. No dependence on the size of the leaving group of ester- and depsipeptide substrates could be observed.

\section{INTRODUCTION}

In previous papers it has been shown that carboxypeptidase $\mathrm{Y}(\mathrm{CPD}-\mathrm{Y})$ is able to catalyze peptide bond formation in aqueous phase $(6,18)$. This reaction proceeds in two steps: A substrate of the enzyme forms an acyl-enzyme intermediate which then undergoes aminolysis in the presence of an amino acid derived nucleophile. An earlier publication from this laboratory concerned the case where the acyl-enzyme intermediate was formed by scission of amide bonds (6). It was demonstrated that peptides and peptide amides are of limited use as substrates in stepwise peptide synthesis. However, the ability of CPD-Y to catalyze the exchange of C-terminal amino acid residues in peptides may become useful in peptide semisynthesis. The present paper deals with CPD-Y catalyzed peptide

\footnotetext{
Abbreviations: $\mathrm{Ac}=$ acetyl; $\mathrm{Bz}=$ benzoyl $\mathrm{CBZ}=$ carbobenzoxy $\mathrm{CPD} \cdot \mathrm{Y}=$ carboxypeptidase $\mathrm{Y} ; \mathrm{Hyac}-\mathrm{OH}$ $=$ hydroxyacetic acid; Hypp-OH $=\alpha$-hydroxy $\beta$-phenyl propionic acid; HPLC $=$ high pressure liquid chromatography; TEAP = triethylammonium phosphate buffer. A mino acids abbreviated in lower case refer to the D-enantiomer. All other abbreviations of amino acids, amino acid derivatives and peptides are according to the guideline of the IUPAC-IUB Commission on Biochemical Nomenclature.
} 
synthesis after scission of the ester bond in ester and depsipeptide substrates, with emphasis on the influence of structural features of the substrate ester on the product yields.

\section{MATERIALS AND METHODS}

\subsection{Materials}

Carboxypeptidase Y from baker's yeast, a commercial preparation of the Carlsberg Breweries, was isolated by a modification of the affinity chromatography procedure of JOHANSEN et al. (13) and obtained as a lyophilized powder (10\% enzyme in sodium citrate). Before use the enzyme was dissolved in water, desalted on Sephadex G-25, fine equilibrated and eluted with distilled water. The concentration of the enzyme was determined spectrophotometrically using $\mathrm{E}_{280 \mathrm{~nm}}^{1 \%}=14.8$. This enzyme preparation was free of Protease A activity as checked by the assay of LeE and RIordan (14).

N-CBZ-L-alanyl-L-alanine methyl ester, Benzoyl-L-alanine methyl ester, Benzoyl-L-alanine, Benzoyl-L-phenylalanine, L-alanyl-L-alanyl-Dalanine, L-alanyl-D-alanyl-L-alanine, Ac- $(\mathrm{Ala})_{\mathrm{n}^{-}}$ OMe ( $\mathrm{n}=1-4)$, Ac-(Ala) $)_{n}-\mathrm{OH}(\mathrm{n}=1-4)$, and L-leucine amide were purchased from Bachem, Liestal, Switzerland. Benzoyl-L-valine methyl ester, Benzoyl-L-leucine methyl ester, BenzoylL-methionine methyl ester, Benzoyl-L-histidine methyl ester, N-CBZ-L-alanyl-L-serine methyl ester, N-CBZ-L-alanyl-L-valine methyl ester, $\mathrm{N}$ CBZ-L-alanyl-L-norvaline methyl ester, N-CBZL-alanyl-L-phenylalanine methyl ester and $\mathrm{N}$ CBZ-L-alanyl-L-tryptophan methyl ester were from Vega-Fox Chemicals, Tucson, USA. Hippuryl-L-phenyllactic acid, Hippuric acid, Lalanyl-L-alanyl-L-alanine methyl ester, glycine, glycine amide $\cdot \mathrm{HCl}$ and $\mathrm{L}$-leucine were obtained from Sigma Chemical Company, St. Louis, USA. N- $\alpha$-Benzoyl-L-arginine methyl ester $\cdot \mathrm{HCl}, \mathrm{N}, \mathrm{N}^{\prime}$-dicyclohexyl carbodiimide and palladium on charcoal (10\% Pd) were obtained from Fluka, Switzerland. Analytical grade solvents and reagents were from Merck, Darmstadt, West Germany and Ferak, Berlin. Hydrogen, hydrogen chloride and 4-(dimethylamino)pyridine for synthesis were from Merck. Triethylamine was from Ferak, Berlin. Benzyl chloroacetate was from ICN Pharmaceuticals, Plainview, N.Y. USA.

\subsection{Synthesis of some ester substrates}

N-Benzoyl-glycyl-hydroxy acetic acid (Bz-GlyHyac-OH) was prepared from hippuric acid and benzyl chloroacetate and subsequent hydrogenolysis of the benzyl ester as described by MURPHY and Bunting (15). N-Benzoyl-phenylalaninehydroxy acetic acid (Bz-Phe-Hyac-OH) was synthesized analogueously using Bz-Phe-OH. The intermediate benzyl ester (Bz-Phe-HyacoBzl) was crystallized from benzene-n-hexane. M.p. was $137-140^{\circ} \mathrm{C}$, yield: $70 \%$. Hydrogenolysis of the benzyl ester gave, after work up, a colorless oil that dispersed in warm petroleum ether $\left(40-60^{\circ} \mathrm{C}\right)$ and then brought into solution by addition of a few drops of 1,2-dichloroethane. Upon cooling the product crystallized as white needles. M.p. was $95-97^{\circ} \mathrm{C}$, yield: $80 \%$. NBenzoyl-phenylalanine methyl ester was prepared as described (5). N-Benzoyl-alanyl benzyl ester was obtained from Bz-Ala-OH and benzyl alcohol according to HASSNER and AleXANian (11). The m.p. was $92-94^{\circ} \mathrm{C}$; Beilstein (4) lists $91{ }^{\circ} \mathrm{C}$ for the racemate. N-Benzoyl-glycyl-methyl ester was prepared by the ion-exchange method (10). The same method was used to prepare the ethyl and isopropyl esters of hippuric acid. The melting points of the products were in agreement with reported values $(7,9)$. N-Benzoyl-glycylbenzyl ester was obtained according to a method reported by SheEhan and Corey (17). H-L-AlaD-Ala-L-Ala-OMe $\cdot \mathrm{HCl}$ and H-L-Ala-L-Ala-DAla-OMe $\cdot \mathrm{HCl}$ were produced from the corresponding tripeptides by repeated esterification with methanol/ $\mathrm{HCl}$ (two times for three hours). The esters were used as oils and were found to be homogeneous by HPLC monitored at $220 \mathrm{~nm}$ (on LiChrosorb RP-18, $10 \mu \mathrm{m}$ using TEAP buffer and acetonitril as eluant (6)).

\subsection{Methods}

Synthesis reactions were performed in a $\mathrm{pH}$ stat. Due to the low solubility of some substrates, several reactions were carried out with the substrate only partly dissolved. The concentrations indicated in the tables are therefore not correct in these cases. At various times aliquots were diluted into $\mathrm{CH}_{3} \mathrm{CN}$ to quench the reaction. The composition of the samples was then determined by HPLC, using reverse-phase support materials and various eluant systems. Those 
Table I

Influence of the C-terminal residue in N-blocked amino acid esters on coupling yields in CPD-Y catalyzed peptide bond formationa).

Substrate

Coupling yield with the following nucleophiles (\%)

\begin{tabular}{lcccc}
\cline { 2 - 5 } & H-Gly-OH & H-Leu-OH & H-Gly-NH & H-Leu-NH \\
\hline Bz-Gly-OMe & 65 & 20 & 60 & 50 \\
Bz-Ala-OMe & 55 & 35 & 90 & 95 \\
Bz-Val-OMec) & 70 & 65 & 85 & $100 \mathrm{~b})$ \\
Bz-Leu-OMec) & 65 & 70 & 95 & 100 \\
Bz-Met-OMec) & 50 & 20 & 95 & 100 \\
Bz-Phe-OMec) & 60 & 25 & 95 & 100 \\
Bz-Tyr-OEtc) & 60 & 70 & 95 & 100 \\
Bz-Arg-OMe & nd & 10 & 95 & 60 \\
Bz-His-OMe & nd & 45 & 95 & 60 \\
\hline
\end{tabular}

a) Conditions: $10 \mathrm{~mm}$-substrate, $0.1 \mathrm{M}-\mathrm{KCl}, 1 \mathrm{~mm}$-EDTA, pH 9.5, CPD-Y $=5-15 \mu \mathrm{M}, 2.0 \mathrm{M}$-glycine, $0.2 \mathrm{M}$ of other nucleophiles, $10 \% \mathrm{CH}_{3} \mathrm{OH}$. Reaction quenched at $5 \mathrm{~min}$.

b) Oligomerization observed, $\mathrm{n}=3$.

c) Reaction carried out with the substrate in suspension.

products not identifiable with parallel runs of relevant standards were collected and the amino acid composition determined. In cases where all compounds of interest had a common dominant chromophore (Bz- and Z-), the concentration could be assessed from the integrated peak areas. When no such dominant chromophore was shared, as in the Ac-(Ala) $)_{n}-\mathrm{OMe}$ series, entire peaks were collected and the concentrations determined by quantitative amino acid analysis. For further details about methods see ref. 6 .

\section{RESULTS}

A systematic investigation was undertaken to determine whether the structure of the side chain of the C-terminal residue in peptide esters could influence the aminolysis/hydrolysis ratio. It is apparent from the data in Table I that the coupling yield for different $\mathrm{N}$-blocked amino acid esters depends strongly on the side group when leucine is used as nucleophile. Using glycine, glycine amide or leucine amide as nucleophile the yields are much more uniform

Table II

Influence of the $\mathrm{C}$-terminal residue in $\mathrm{N}$-blocked dipeptide esters on coupling yields in CPD-Y catalyzed peptide bond formationa).

Substrate

Coupling yield with the following nucleophiles $(\%)$

\begin{tabular}{lcc}
\cline { 2 - 3 } & H-Leu-OH & H-Leu-NH \\
\hline Z-Ala-Ala-OMe & 0 & 100 \\
Z-Ala-Ser-OMe & 40 & 75 \\
Z-Ala-Val-OMeb) & 25 & 100 \\
Z-Ala-Nvl-OMeb) & 15 & 100 \\
Z-Ala-Phe-OMeb) & 0 & 90 \\
Z-Ala-Trp-OMeb) & 5 & 90 \\
\hline
\end{tabular}

a) Conditions: 10mm-substrate, $0.1 \mathrm{~m}-\mathrm{KCl}, 1 \mathrm{~mm}-\mathrm{EDTA}, \mathrm{pH} 9.5 \mathrm{CPD}-\mathrm{Y}=5 \mu \mathrm{M}, 0.15 \mathrm{~m}-$ nucleophile, $10 \%$ $\mathrm{CH}_{3} \mathrm{OH}$. Reaction quenched at $20 \mathrm{~min}$.

b) Reaction carried out with substrate in suspension. 
Table III

Influence of the length of the peptide ester on coupling yields in CPD-Y catalyzed peptide bond formationa).

Substrate

Coupling yields with the following nucleophiles (\%)

\begin{tabular}{lcc}
\cline { 2 - 3 } & H-Leu-OH & H-Leu-NH 2 \\
\hline Ac-Ala-OMe & 50 & 90 \\
Ac-(Ala) 2 -OMe & 20 & 80 \\
Ac-(Ala) $)_{3}$-OMe & 5 & 90 \\
Ac-(Ala) $_{4}-\mathrm{OMe}$ & $<5$ & 90 \\
\hline
\end{tabular}

a) Conditions: See Table II, except that Ac-(Ala) 4 $_{4}$-OMe reactions are quenched at $10 \mathrm{~min}$.

\section{Table IV}

Influence of the chirality of the substrate on the coupling yields in CPD-Y catalyzed peptide bond formationa).

\begin{tabular}{lcc}
\hline Substrate & Product & Yield (\%) \\
\hline H-Ala-Ala-Ala-OMe & H-Ala-Ala-Ala-Leu- $\mathrm{NH}_{2}$ & 80 \\
H-Ala-ala-Ala-OMe & H-Ala-ala-Ala-Leu-NH2 & $40 \mathrm{~b})$ \\
H-Ala-Ala-ala-OMe & H-Ala-Ala-ala-Leu-NH2 & $0 \mathrm{c})$ \\
\hline
\end{tabular}

a) Conditions: $25 \mathrm{~mm}$-substrate, $0.1 \mathrm{~m}-\mathrm{KCl}, 1 \mathrm{~mm}$-EDTA, pH 9.5, CPD-Y $=12 \mu \mathrm{M}, 0.2 \mathrm{~m}$-leucine amide, reaction quenched at $20 \mathrm{~min}$.

b) Conversion of this ester was approximately 10 times slower than for the L-analogue.

c) This ester was not converted under the conditions in a).

Table V

Influence of the substrate ester leaving group on coupling yields in CPD-Y catalyzed peptide bond formation.

\begin{tabular}{lcccc}
\hline \multirow{2}{*}{ Substrate } & \multicolumn{4}{c}{ Coupling yield with the following nucleophiles (\%) } \\
\cline { 2 - 5 } & H-Gly-OH & H-Leu-OH & H-Gly-NH & H-Leu-NH 2 \\
\hline Bz-Gly-OMea) & 65 & 65 & 60 & 55 \\
Bz-Gly-OEta) & 55 & 80 & 95 & 95 \\
Bz-Gly-OiPra) & 45 & 85 & 85 & 95 \\
Bz-Gly-OBzla, c) & 45 & 70 & 90 & 95 \\
Bz-Ala-OMea) & 40 & 25 & 90 & 90 \\
Bz-Ala-OBzla, c) & 30 & 20 & 90 & 90 \\
Bz-Gly-Hyac-OHb) & - & - & 90 & 95 \\
Bz-Phe-Hyac-OHb) & - & - & 90 & 90 \\
Bz-Gly-Hypp-OHb) & - & - & 95 & 95 \\
\hline
\end{tabular}

a) Conditions: See Table I, except quenched at $20 \mathrm{~min}$.

b) Like a) except $\mathrm{pH} 7.5$.

c) Reaction carried out with substrate in suspension. 
and generally higher. Variations in the Cterminal residue of $\mathrm{N}$-blocked dipeptide esters lead to similar results (Table II). While the use of leucine as nucleophile revealed a strong dependence of the yields on the $\mathrm{C}$-terminal residue of the ester substrate, no such influence of the substrate structure was evident with leucine amide as the nucleophile.

Although the data in Table I and II indicated that elongation of the peptide chain in the ester substrate resulted in lower yields with leucine as nucleophile, additional experiments were performed to study this dependence. With the substrate series Ac-(Ala) ${ }_{n}-\mathrm{OMe}, \mathrm{n}=1-4$ it was documented that the longer esters were characterized by very low coupling yields with leucine (Table III). Going from $n=1$ to $n=4$ the yield decreased from $50 \%$ to $5 \%$. However, using leucine amide as nucleophile the yield remained constant around $90 \%$, independent of the chain length.

In Table IV it is demonstrated that the absence of an N-blocking group does not seem to affect the coupling yield, since H-Ala-Ala-Ala-OMe gives approximately the same yield with leucine amide as Ac-Ala-Ala-Ala-OMe (Table III). It is furthermore shown that if the C-terminal residue is present in its D-enantiomer as in $\mathrm{H}$ Ala-Ala-ala-OMe no peptide synthesis is obtained due to lack of turnover of the substrate. With the amino acid at the penultimate position in the D-configuration as in H-Ala-ala-Ala-OMe a turnover can be registered, but the coupling yield with leucine amide is reduced (40\%) compared to the yield with H-Ala-Ala-Ala-OMe ( $80 \%$ ).

It was conceivable that the aminolysis/ hydrolysis ratio could be influenced by the size of the ester leaving group. The results in Table $\mathrm{V}$ indicate this is not the case. Going from a methyl to a benzyl ester insignificant changes in yields are observed. This is true for both the Bz-Alaand the Bz-Gly-series. Varying the size of the leaving group of depsipeptides lead to the same conclusion, since Bz-Gly-Hyac-OH with leucine amide resulted in the same coupling yield as $\mathrm{Bz}$ Gly-Hypp-OH (Table V). The reactions with the depsipeptides were all performed at $\mathrm{pH} 7.5$ due to the lack of turnover at $\mathrm{pH} 9.5$. At $\mathrm{pH} 7.5$ it is not possible to use amino acids as nucleophiles, since the products (peptides) are rapidly hydrolyzed.

\section{DISCUSSION}

In order for CPD-Y to successfully catalyze the formation of peptide bonds, an acyl-enzyme intermediate must be formed (8). In addition, the amino acid or amino acid derivative used as a nucleophile must bind to the enzyme at a specific location for the nucleophilic attack on the acylenzyme intermediate. Considering that at $\mathrm{pH}$ $>9$ the amidase activity of CPD-Y is low and the carboxypeptidase activity is minimal relative to the esterase activity, the most obvious approach using CPD-Y in peptide synthesis, was to use peptide esters as substrates and amino acids or amino acid amides as nucleophiles (18). The present paper further explores this approach with special emphasis on effects of varying the composition of the substrate.

Several factors could conceivably affect the aminolysis/hydrolysis ratio in a synthesis reaction with peptide esters and various nucleophiles. Since the CPD-Y catalyzed peptide bond formation probably takes place while both the acyland amine-donating portions interact with the enzyme, the coupling must depend on these interactions. However, varying the nature of the substrate does not in all cases lead to different coupling yields with a given nucleophile. The reactions of $\mathrm{Bz}-\mathrm{X}$-OMe with leucine amide, glycine amide and glycine seem less dependent on the nature of $\mathrm{X}$ than is the case with leucine (Table I). This result can be rationalized, when considering that leucine contains a negative charge and a bulky side group as well. Both factors may result in modes of binding that are not optimal for nucleophilic attack. Conversely, the three other nucleophiles either lack the bulky side group or the negative charge.

In order for CPD-Y to exhibit esterase activity, the C-terminal amino acid must be bound in the $S_{1}$ site (Berger and Schechter nomenclature (16)). For other proteolytic enzymes the influence of 5-7 subsites on the binding of peptides has been described $(1,2,16,19)$. It was thus important to establish whether interactions at other sites influenced the coupling yields. The results in Table II indicate that also with longer substrates are the coupling yields, using leucine as nucleophile, very dependent on the nature of the $\mathrm{C}$-terminal amino acid residue. The effects of the length of the peptide chain on the coupling yield with leucine as nucleophile (Table 
III) should be judged cautiously, since these effects need not originate from chain-elongation as such but rather from unfavourable subsite interactions.

The presence of D-amino acids in the peptide ester substrates forms another aspect of the influence of substrate-enzyme interactions on the yield of aminolysis. It had already been documented that $\mathrm{N}$-blocked $\mathrm{D}$-amino acid esters are not substrates of CPD-Y (3) and neither is AlaAla-ala-OMe (Table IV). However, with Ala-alaAla-OMe a slow turnover could be registered, and coupling in the presence of leucine amide as well. Interestingly, the yield was only half of that obtained with Ala-Ala-Ala-OMe. This result gives further evidence for the influence of subsite interactions on the yield of aminolysis. Additionally, it may also be pointed out, that the lack of an N-blocking group on a tripeptide ester influences the rate of hydrolysis compared to the corresponding acetylated species, whereas the yield of aminolysis, in the presence of leucine amide, is not affected. Unblocked dipeptide esters are hydrolyzed by the enzyme but nonenzymatic formation of diketopiperazines forms in most cases an obstacle to the use of such substrates in this type of peptide synthesis.

The sequence of binding of substrate and nucleophile to the enzyme is a question that cannot unequivocally be answered in the present study. Is the nucleophile bound to the enzyme while acylation is taking place, or does it bind to the enzyme after the acylation and expulsion of the leaving group? In both cases the competition between nucleophile and water for the acylenzyme could be dependent on the characteristics of the leaving group of the substrate. It has already been documented for CPD-Y catalyzed transpeptidations that the size of the leaving group (an amino acid) is important for the yield of the aminolysis reaction (6). No such dependence on size could be documented with an alcohol or a hydroxyacid leaving group as in peptide esters or depsipeptides, respectively (Table V). The difference between the two groups of substrates is clearly manifested in the following example: With leucine amide as nucleophile and $\mathrm{Bz}-\mathrm{Gly}-\mathrm{Hypp}-\mathrm{OH}$ as substrate the yield of Bz-Gly-Leu- $\mathrm{NH}_{2}$ is $95 \%$, whereas for the corresponding reaction using $\mathrm{Bz}$-GlyPhe- $\mathrm{OH}$ as substrate the yield is only $10 \%$. The two substrates only differ in the nature of the scissile bond, one being an amide bond, the other an ester bond. It can be concluded, regardless of which of the two binding models mentioned above is correct, that when large amino acids serve as leaving groups, the nucleophile is impeded in its attack on the acyl-enzyme. For esters and depsipeptides the attack of the nucleophile is not restricted by the size of the leaving group. The high rotational freedom around an ester bond compared to an amide bond, and/or a faster expulsion of the leaving group from the active site, could account for these differences.

The fact that increasing the size of the ester leaving group increases the rate of disappearance of substrate while not affecting the yield of aminolysis can be very useful in synthesis reactions. For certain amino acid sequences the peptide amide formed in a coupling reaction can be a better substrate than the original ester substrate. This results in undesirable side reactions, but the ability of being able to increase the rate of disappearance of the ester substrate, and thereby decrease the relative reactivity of the product, can help minimizing these effects. It can also be important in such cases to evaluate the influence of the solubility of reactants and product(s) on the reaction course. In some instances the rate of hydrolysis of the substrate may be low due to its low solubility, while the rate of hydrolysis of the product is high due to its high solubility. The reaction between $\mathrm{Bz}-\mathrm{Val}$ $\mathrm{OMe}$ and leucine amide is such a case. An oligomerization is observed due to the higher solubility of $\mathrm{Bz}$-Val-Leu- $\mathrm{NH}_{2}$ relative to $\mathrm{Bz}-\mathrm{Val}$ OMe (Table I). It must be pointed out that the reaction time can influence the product composition too.

Taken in conjunction with the earlier publication dealing with the use of peptides and peptide amides (6) the results in this paper demonstrate the wide variety of substrates that can be used in CPD-Y catalyzed peptide synthesis. The conclusion from these studies is that in stepwise peptide synthesis, $\mathrm{N}$-blocked ester substrates and amino acid amide nucleophiles are to be preferred in general. However, the need for deamidation of the peptide amide product before reesterification could in some cases make the direct coupling with amino acids advantageous. The ability of 
CPD-Y to hydrolyse unblocked peptide esters, and peptide esters with D-amino acids in the penultimate position can be useful for the synthesis of certain peptides. This is also true for the use of peptide amides as substrates. In establishing a synthetic scheme it must be recommended to test many possibilities for the use of substrates and nucleophiles in order to obtain an overall optimal yield of synthesis.

\section{ACKNOWLEDGEMENTS}

The authors are deeply indepted to Professor MarTin OTTESEn for many helpful suggestions at all stages of the work. The excellent technical assistance of Mss. Bodil Corneliussen, IRENE Simonsen and Edith Fløistrup is gratefully acknowledged. F. WIDMER gratefully acknowledges the Swiss National Science Foundation for a Post-Doctoral Fellowship.

\section{REFERENCES}

1. Abramowitz, N., I. Schechter \& A. Berger: On the size of the active site in proteases. II. Carboxypeptidase A. Biochem. Biophys. Res. Commun. 29, 862-867 (1967)

2. Atlas, D. \& A. Berger: On the specificity of elastase. Hydrolysis of peptide p-nitrobenzylesters. Biochemistry 11, 4719-4723 (1972)

3. Bal, Y, R. Hayashi \& T. Hata: Kinetic studies of Carboxypeptidase Y. III. Action on ester, amide, and anilide substrates and the effects of some environmental factors. J. Biochem. (Tokyo) 77, 617-636 (1975)

4. Beilstein Handbuch der Organischen Chemie, 3. Erg-Werk, Bd. IX/2, 1143. Springer-Verlag, Berlin (1970)

5. Bergmann, M. \& L. Zervas: A method for the stepwise degradation of polypeptides. J. Biol. Chem. 113, 341-357 (1936)

6. Breddam, K., F. Widmer \& J. T. Johansen: Carboxypeptidase Y catalyzed transpeptidations and enzymatic peptide synthesis. Carlsberg Res. Commun. 45, 237-247 (1980)
7. Epand, R. \& J. Wilson: Evidence for the formation of hippuryl chymotrypsin during the hydrolysis of hippuric acid esters. J. Biol. Chem. 238, 1718-1723 (1963)

8. Fastrez, J. \& A. R. Ferscht:Demonstration of the acyl-enzyme mechanism for the hydrolysis of peptides and anilides by chymotrypsin. Biochemistry 12, 2025-2034 (1973)

9. Franzen, H.: Über Acylieren von Aminen. Chem. Ber. 42, 2465-2468 (1909)

10. Hammond, B. R. \& H. Gulfreund: The mechanism of ficin-catalyzed reactions. Biochem. J. 72, 349-357 (1959)

11. Hassner, A. \& V. Alexanian: Direct room temperature esterification of carboxylic acids. Tetrahedron Lett., pp. 4475-4478 (1978)

12. Hayashi, R., Y. BaI \& T. HaTa: Kinetic studies of carboxypeptidase Y. I. Kinetic parameters for hydrolysis of synthetic substrates. J. Biochem. (Tokyo) 77, 69-79 (1975)

13. Johansen, J. T., K. Breddam \& M. Ottesen: Isolation of carboxypeptidase $\mathrm{Y}$ by affinity chromatography. Carlsberg Res. Commun. 41, $1-14(1976)$

14. LeE, H.-M. \& J. F. Riordan: Does carboxypeptidase $\mathrm{Y}$ have intrinsic endopeptidase activity. Biochem. Biophys. Res. Commun. 85, $1135-$ 1142 (1978)

15. Murphy, J. \& J.W. Bunting: Substrate activation in the carboxypeptidase A catalysis of ester hydrolysis. Can. J. Chem. 52, 3829-3836 (1974)

16. Schechter, I. \& B. Berger: On the size of the active site of proteases. I. Papain, Biochem. Biophys. Res. Commun. 27, 157-162 (1967)

17. Shefhan, J. C. \& E. J. Corey:Synthesis and reactions of acyclic N,N-diacylglycines. J. Am. Chem. Soc. 74, 4555-4559 (1952)

18. WIDMER, F. \& J. T. JOHANSEN: Enzymatic peptide synthesis. Carboxypeptidase $Y$ catalyzed formation of peptide bonds. Carlsberg Res. Commun. 44, 37-46 (1979)

19. Үамамото, T. \& N. Izumiya: Action of trypsin on synthetic substrates, III. Hydrolysis of homologous peptides ranging from diglycyl-L. lysyl-glycin to diglycyl-L-lysyl-tetraglycine. Arch. Biochem. Biophys. 120, 497-502 (1967) 